\title{
BIOPSIASIN ASPIRACIÓN CON AGUJA FINA ECOGUIADA TRANSABDOMINAL EN EL DIAGNÓSTICO DE NEOPLASIAS PROSTÁTICAS EN CANINOS
}

\section{Transabdominal Echo-guided Fine Needle Biopsy without Aspiration in the Diagnosis of Canine Prostatic Neoplasia}

\author{
Ricardo Grandez R. ${ }^{1,3}$, Claudia Miguel de Priego G. ${ }^{1}$, Javier Mamani P. ${ }^{2}$, \\ Christian Pitot A. ${ }^{2}$
}

\section{Resumen}

La biopsia por aspiración con aguja fina ecoguiada transabdominal es una técnica segura y mínimamente invasiva utilizada para confirmar el diagnóstico de neoplasia prostática. Una variación de esta técnica es la biopsia sin aspiración con aguja fina (BSAAF) donde no se ejerce presión negativa para obtener el material. Cuatro casos con alteraciones clínicas y ultrasonográficas compatibles con neoplasia prostática fueron evaluados con la técnica BSAAF ecoguiada. El procedimiento fue realizado con la guía de un ultrasonógrafo convencional. En las áreas de interés para la colección de la mues-

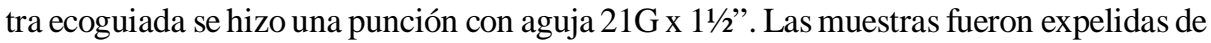
la aguja con una jeringa sobre una lámina de vidrio y teñidas con Tinción 15®. La interpretación de las muestras estuvo basada en los criterios de identificación de células neoplásicas. La técnica BSAAF ecoguiada permitió obtener suficiente cantidad de material biológico con una mínima contaminación de sangre, mínima formación de artefactos, y una apropiada conservación de la morfología celular para realizar una adecuada interpretación y diagnóstico citológico de neoplasias prostáticas.

Palabras clave: canino, próstata, biopsia ecoguiada, neoplasia

\section{AbSTRACT}

Fine-needle aspiration biopsy guided with trans-abdominal ultrasound is a safe and minimally invasive technique that is used to confirm the diagnosis of prostatic neoplasia. A variation of this technique is a fine-needle biopsy without aspiration in which negative pressure is not exerted when obtaining the material. Four cases with clinical and ultrasound alterations compatible with prostatic neoplasia were evaluated with ultrasound-guided fine-needle biopsy without aspiration as a diagnostic tool. The procedure was done with

\footnotetext{
${ }^{1}$ Clínica Veterinaria Cayetano Heredia, ${ }^{2}$ Laboratorio de Histología y Patología, Facultad de Veterinaria y Zootecnia, Universidad Peruana Cayetano Heredia, Lima

${ }^{3}$ E-mail: ricardo_grandez@upch.pe
} 
conventional ultrasound guidance. When the area of interest was found, an ultrasoundguided sample collection was performed using a $21 \mathrm{G}$ x $1 \frac{1}{2}$ " needle. The sample was expelled through the needle with a syringe onto a glass slide and stained using Tinción $15 \circledR$. Sample interpretation was based on the identification of tumor cells and general criteria of malignancy. Ultrasound-guided fine-needle biopsy without aspiration as a diagnostic tool allowed obtaining sufficient quantity of biological material with minimal blood contamination and formation of artifacts, and appropriate conservation of cellular morphology to perform suitable interpretation and cytological diagnosis of prostatic neoplasia.

Key words: canine, prostate, echo-guided biopsy

\section{INTRODUCCIÓN}

La próstata es la única glándula sexual accesoria en el canino. Está localizada caudal a la vejiga, es encapsulada, bilobulada y rodea a la uretra proximal (Evans y de Lahunta, 1997; Boyd y Paterson, 2001). Su posición depende de la edad, de la relajación de la vejiga y de los cambios fisiológicos que sufre la glándula.

La enfermedad prostática es una condición patológica que contribuye a la presentación de signos clínicos gastrointestinales como constipación y tenesmo; aunque en algunos casos puede estar asociada con disfunción del tracto genitourinario (Ruel $e t$ al., 1998; Stornelli et al., 2002). Los desórdenes más comunes son la hiperplasia prostática benigna (HPB), prostatitis bacteriana, quistes, abscesos y neoplasias prostáticas (Krawiec y Heflin, 1992; Atalan et al., 1999; Prüfer et al., 2000; White, 2000).

La prevalencia de neoplasias prostáticas a la necropsia en el canino, sin signos clínicos previos de enfermedad prostática es de 3.6\% (Mukatatirwa y Chitura, 2007), mientras que la prevalencia de neoplasias prostáticas con signos clínicos es de $13.0 \%$ (Teske et al., 2002). La forma de neoplasia más común es el adenocarcinoma, pero también se han descrito patologías como carcinoma indiferenciado, carcinoma de células transicionales, leiomiosarcoma y carcinoma de células escamosas (Swinney, 1998). En el examen clínico, los caninos con cáncer prostático presentan prostatomegalia y signos clínicos similares a otras enfermedades del tracto gastrointestinal o genitourinario como tenesmo, constipación, disquesia, disuria, hematuria, incontinencia urinaria y, eventualmente, signos sistémicos o locomotores (Swinney, 1998; Foster, 2000; Tyler et al., 2003).

El tacto rectal y la radiología tienen limitaciones para el diagnóstico de neoplasias prostáticas, dado que no se puede precisar el tamaño ni el detalle de la arquitectura prostática (Johnston et al., 2000; Kamolpatana et al., 2000; Gonzáles, 2004). Por otro lado, la ultrasonografía abdominal es una técnica simple y segura para el paciente, que provee información dinámica e inmediata de la arquitectura prostática, así como la extensión y ubicación de las posibles lesiones (Prüfer et al., 2000; Guido, 2004).

Con el fin de obtener un diagnóstico más certero de las neoplasias prostáticas se requiere el uso de técnicas complementarias como la Biopsia por Aspiración con Aguja Fina (BAAF) (Hörauf y Tamke, 2000; Thomas et al., 2002). Cuando la BAAF es guiada mediante ultrasonografía transabdominal se constituye en una técnica de biopsia segura y mínimamente invasiva (Maffrand et al., 2007). La Biopsia sin Aspiración con Aguja Fina (BSAAF) es una variante de la $\mathrm{BAAF}$, que no requiere ejercer una presión 
negativa para obtener la muestra, y está indicada en citologías de tejidos fuertemente vascularizados (Tyler et al., 2003; Nyland $e t$ al., 2004).

El presente estudio presenta cuatro casos de pacientes con alteraciones ultrasonográficas compatibles con neoplasia prostática y evalúa la técnica de BSAAF ecoguiada transabdominal como herramienta diagnóstica.

\section{MateRIALES Y Métodos}

\section{Casos Clínicos}

Cuatro pacientes caninos que presentaban signos clínicos de enfermedad prostática, presumiblemente neoplásicas, no relacionados entre sí, fueron referidos al Servicio de Diagnóstico por Imágenes de la Clínica Veterinaria Cayetano Heredia, en Lima, Perú, entre enero de 2009 y julio de 2012. Se les realizó ultrasonografía abdominal convencional, encontrando en todos ellos alteraciones ultrasonográficas compatibles con neoplasia prostática. Se recomendó realizar la técnica de BSAAF ecoguiada y el estudio citológico respectivo para determinar el tipo de patología presente.

Caso 1 (C1): Canino, Bóxer macho entero de 8 años de edad, con historia de anorexia, disuria, constipación y tenesmo. El examen físico general reveló una buena condición corporal y pelaje con moderada descamación. Durante la palpación abdominal, la vejiga estuvo relajada y se pudo palpar una masa consistente en la región pélvica.

Caso 2 (C2): Canino, Setter Irlandés macho entero de 7 años de edad, con historia de constipación, melena y ligera anorexia. El examen físico general reveló buena condición corporal y pelaje brillante. Se pudo palpar una masa en el abdomen bajo.
Caso 3 (C3): Canino, de raza cruzada, macho entero de 11 años de edad, con historia de tres meses de constipación, disuria, sangrado prepucial, depresión y anorexia. El examen físico general indicó depresión, pelaje hirsuto y pobre condición corporal.

Caso 4 (C4): Canino, Siberian Husky macho entero de 5 años de edad, con historia de sangrado prepucial, anorexia, anuria, depresión, constipación y diarrea oscura en los días previos. El examen físico general mostró emaciación, ictericia y pelaje hirsuto. Se identificó en la base del pene una masa tumoral proliferativa compatible con tumor venéreo transmisible. Se tomaron muestras por impresión para citología diagnóstica.

\section{Biopsia sin Aspiración con Aguja Fina (BSAAF) Ecoguiada}

Los animales fueron sedados y posicionados en decúbito dorsal. La evaluación de la próstata fue realizada con el uso de un ultrasonógrafo Pie Medical 100 Falco con transductor dual multifrecuencial de 57.5 MHz para determinar el tamaño, forma, ecogenicidad y ecotextura de la próstata e identificar alteraciones ultrasonográficas prostáticas consistentes con una patología.

Una vez localizadas las áreas de interés, se procedió con la técnica de BSAAF ecoguiada usando agujas de $21 \mathrm{G}$ x 11/2". La técnica se realizó adaptando los procedimientos descritos en la literatura (Morris y Dobson, 2002; Thomas et al., 2002; Nyland et al., 2004). La aguja fue parcialmente introducida vía transabdominal dentro del tejido prostático y luego, guiada por la imagen ultrasonográfica, fue totalmente introducida para tomar muestras del área de interés. Se hicieron entre 8 a 10 movimientos de avance y retroceso, a la vez que se redirigía la aguja para colectar células por efecto de corte y presión sobre el tejido. Las muestras con presencia de contaminación de sangre en el cono de la aguja fueron descartadas. 


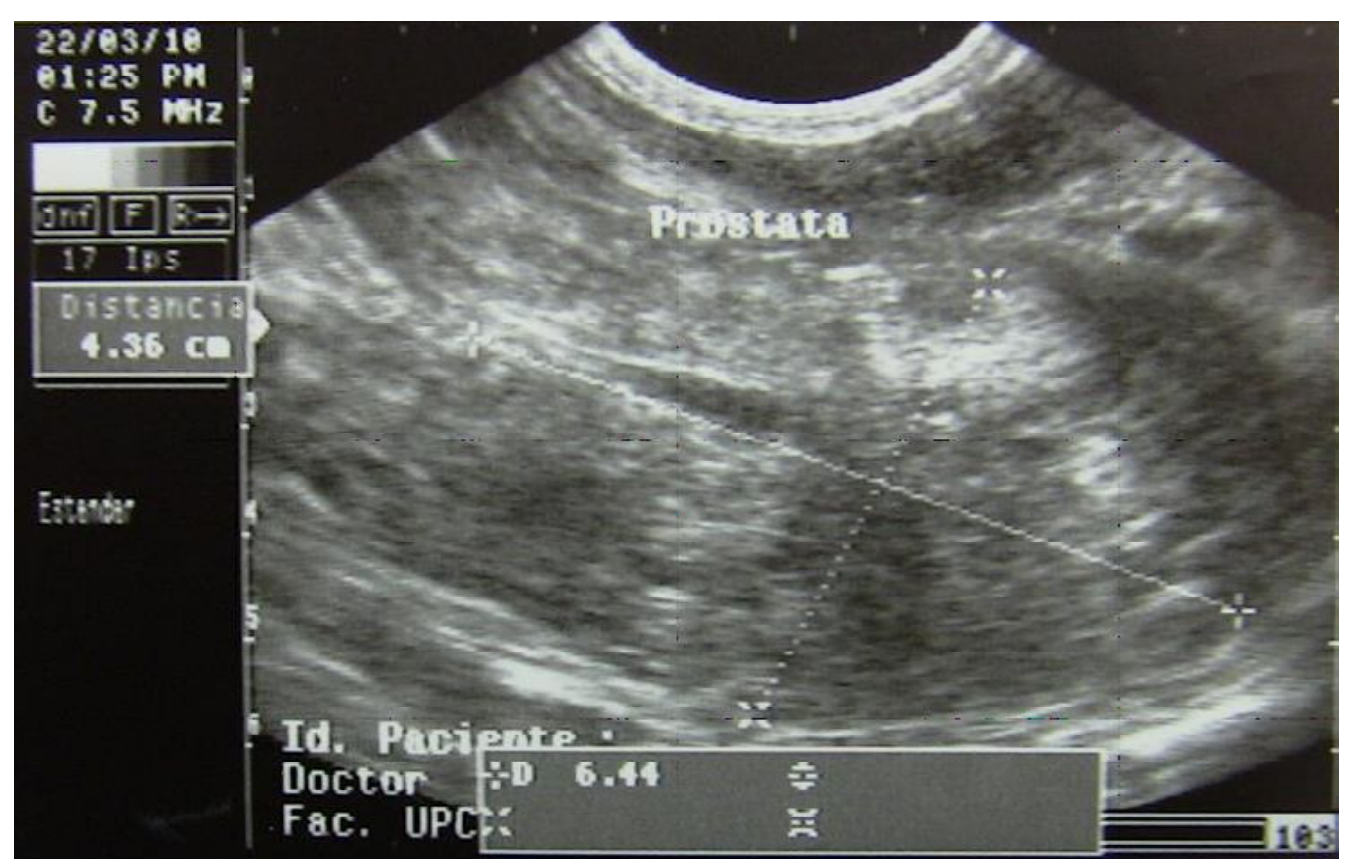

Figura 1. Ultrasonografía abdominal de canino (Caso 2) con alteraciones compatibles con neoplasia prostática. Se observa la prostatomegalia $(6.4$ x $4.3 \mathrm{~cm})$ de bordes imprecisos y discretamente discontinuos y la ecotextura heterogénea con áreas hipoecogénicas irregulares

\section{Citología}

Las muestras fueron expelidas de la aguja con ayuda de una jeringa con aire. Una gota del material biológico obtenido fue depositado sobre una lámina de vidrio y distribuido de acuerdo a técnicas citológicas combinadas (Tyler et al., 2003). Las extensiones fueron teñidas con Tinción $15 \AA$ y evaluadas en el Laboratorio de Histología y Patología de la Facultad de Veterinaria y Zootecnia, Universidad Peruana Cayetano Heredia, siguiendo los criterios de características citológicas de la población celular, pleomorfismo y atipia; características celulares de tamaño, diferenciación y relación núcleo citoplasmática; y características nucleares de tamaño, forma, número, cromaticidad y número de núcleos y nucléolos (Belford y Lumsden, 2000; Morris y Dobson, 2002; Tyler et al., 2003).

\section{Resultados}

La técnica de BSAAF ecoguiada permitió, en los cuatro casos, realizar una adecuada evaluación, establecer dimensiones y características ecográficas de las próstatas afectadas; así como seleccionar las áreas lesionadas y obtener el material biológico suficiente para realizar un apropiado diagnóstico citológico de las neoplasias prostáticas.

C1 mostró prostatomegalia $(6.4 \times 5.3$ $\mathrm{cm})$, ecotextura heterogénea de forma irregular y con áreas anaecoicas (estructuras cavitarias) compatibles con grandes quistes. Los riñones mostraron cambios ultrasonográficos compatibles con hidronefrosis. En las muestras colectadas se observó grupos de células cúbicas de citoplasma granular, vacuolas redondeadas, granulares y, en algu- 


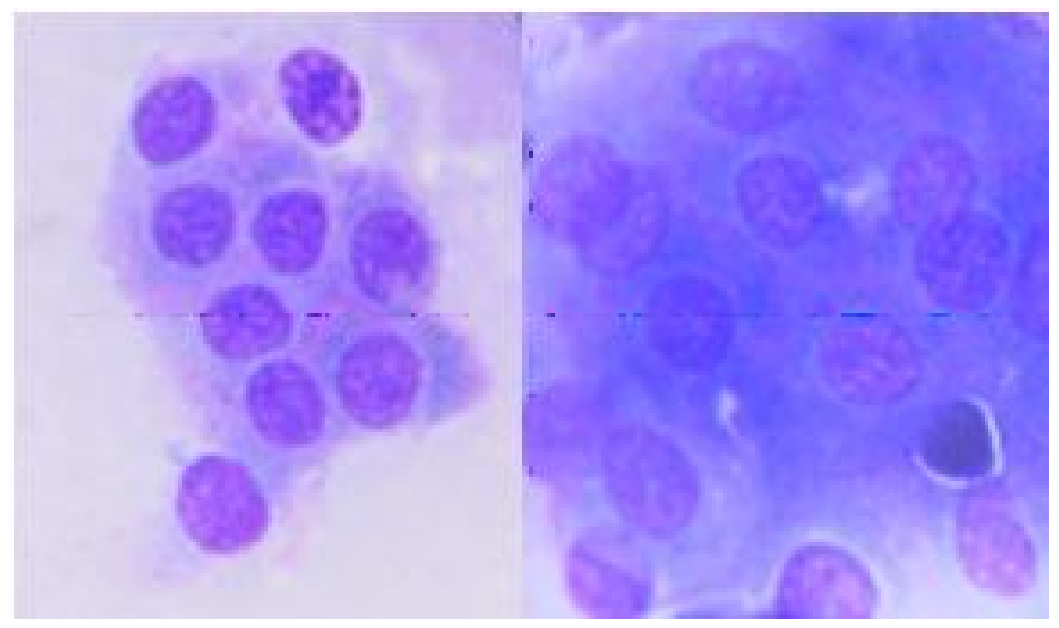

Figura 2. Microfotografía de extendido prostático de canino (Caso 4) obtenido mediante biopsia sin aspiración con aguja fina ecoguiada. Se observan numerosas agrupaciones de células poliédricas bien delimitadas con variable cantidad de citoplasma basofílico, ligeramente granular; núcleos redondeados de bordes irregulares y con cromatina en grumos, así como marcada anisocitosis, anisocariosis, pérdida de la relación núcleo/ citoplasma, y presencia de 2-4 nucléolos prominentes por núcleo, compatible con neoplasia de células epiteliales de características malignas

nos casos, con muescas, de uno a tres nucléolos voluminosos, relación núcleo citoplasma incrementado y moderada anisocitosis y anisocariosis. El extendido fue compatible con una neoplasia de células epiteliales de características malignas.

C2 mostró prostatomegalia $(6.4 \times 4.3$ $\mathrm{cm})$ de bordes imprecisos, discretamente discontinuos y de ecotextura heterogénea, con áreas hipoecogénicas irregulares y sin evidencia de áreas cavitarias (Fig. 1). La citología presentó células redondeadas con citoplasma ligeramente basófilo, formando grupos con núcleos redondeados con dos a tres nucléolos prominentes, mitosis anormales, relación núcleo citoplasma incrementada, así como presencia de eritrocitos y células epiteliales exfoliadas en varios estados de autolisis. El extendido fue compatible con una neoplasia de células epiteliales de características malignas.
C3 presentó prostatomegalia $(7.2 \times 5.6$ $\mathrm{cm})$ de forma irregular con áreas anaecoicas compatibles con quistes y con parénquima de ecotextura heterogénea. Los riñones mostraron cambios ultrasonográficos compatible con hidronefrosis. En la evaluación microscópica se observó células epiteliales con citoplasma ligeramente basofílico y con dos a tres nucléolos redondeados, moderada anisocitosis y anisocariosis, relación núcleo citoplasma incrementada y figuras de mitosis anormales. El extendido fue compatible con una neoplasia de células epiteliales de características malignas.

C4 presentó una evidente prostatomegalia $(8.9 \times 5.5 \mathrm{~cm})$ con bordes irregulares y de ecotextura heterogénea, y algunas áreas hiperecogénicas compatibles con mineralización. La citología mostró numerosas agrupaciones de células poliédricas bien delimitadas con variable cantidad de citoplas- 
ma basofílico ligeramente granular, núcleos redondeados de bordes irregulares y con cromatina en grumos. Asimismo, había marcada anisocitosis, anisocariosis y pérdida de la relación núcleo/citoplasma, y presencia de 2 a 4 nucléolos prominentes por núcleo. El extendido fue compatible con una neoplasia de células epiteliales de características malignas (Fig. 2).

\section{Discusión}

Las casos incluidos en este reporte corresponden a animales adultos y gerontes, de tamaño mediano a grande, no castrados y con signos de enfermedad prostática que corresponde a los descritos en estudios previos (Johnston et al., 2000; Gobello et al., 2002; Teske et al., 2002; Tyler et al., 2003). Todas las evaluaciones ultrasonográficas mostraron lesiones características consistentes con neoplasias prostáticas (Prüfer et al., 2000; Guido, 2004; Nyland y Mattoon, 2004). Adicionalmente, se observó hidronefrosis (Cotran et al., 1995) en dos casos como consecuencia de la disrupción del fluido urinario normal causadas por las masas de las neoplásicas prostáticas. Estos caninos $(\mathrm{C} 1$, C2) mostraron signos clínicos de enfermedad del tracto genitourinario como son disuria, tenesmo y sangrado prepucial. También se observó un caso (C4) de tumor venéreo transmisible, patología frecuente en perros vagabundos sexualmente activos (Ortega et al., 2003).

La técnica de BSAAF ecoguiada demostró ser un procedimiento adecuado para el diagnóstico de neoplasias prostáticas, permitiendo un fácil acceso, selección de áreas de lesión (incluyendo las estructuras cavitarias), y toma de muestras de las próstatas afectadas (Kanayama, 2004). Mediante esta técnica fue posible realizar una toma de muestra representativa de la lesión con el uso de una mano, mientras con la otra se controlaba el transductor ultrasonográfico, permitiendo una colocación precisa de la aguja
(Hörauf y Tamke, 2000; Nyland et al., 2004). La técnica también requirió un mínimo de trabajo para obtener un suficiente número de muestras con baja contaminación de sangre periférica y preservando la morfología celular; permitiendo una apropiada evaluación y diagnóstico citológico, como se describe en la literatura consultada (Powe et al., 2004), a pesar de la ausencia del uso de presión negativa durante la realización del procedimiento.

Aunque la implantación neoplásica por la técnica de BSAAF es una complicación rara, puede ser reducida con el uso de agujas de pequeño calibre, $21 \mathrm{G}$ o menores (Thomas et al., 2002). Si bien la implantación constituye una seria complicación en el largo término, no debe influenciar en la decisión de realizar esta técnica, debido a que los beneficios exceden a los riesgos en el corto término.

En conclusión, la técnica de BSAAF guiada con ultrasonografía permite localizar las áreas de lesión y obtener una cantidad suficiente de material biológico con escasa contaminación de sangre periférica, mínima formación de artefactos y una adecuada conservación de la morfología celular para realizar una apropiada interpretación y diagnóstico citológico en próstatas neoplásicas.

\section{Literatura Citada}

\section{Atalan G, Holt PE, Barr FJ, Brown} PJ.1999. Ultrasonographic estimation of prostatic size in canine cadavers. Res Vet Sci 67: 7-15.

2. Boyd JS, Paterson C. 2001. Color atlas of clinical anatomy of the dog and cat. $2^{\text {nd }}$ ed. London: Mosby. 218 p.

3. Belford C, Lumsden J. 2000. Citopatología. En: Davidson M, Else R, Lumsden J (eds). Manual de patología clínica en pequeños animales. Madrid: Harcourt. p 169-193.

4. Cotran RS, Kumar V, Robbins SL. 1995. Aparato genital masculino. En: Schoen FJ (ed). Patología estructural y 
funcional. Madrid: McGraw-Hill Interamericana. p 1130-1132.

5. Evans $H$, de Lahunta A.1997. Disección del perro. $4^{\text {th }}$ ed. México DF: McGraw-Hill Interamericana. 365 p.

6. Foster RA. 2000. Sistema reproductor. En: Davidson M, Else R, Lumsden J (eds). Manual de patología clínica en pequeños animales. Madrid: Harcourt. p 459-479.

7. Gobello C, Castex G, Corrada Y. 2002. Serum and seminal markers in the diagnosis of disorders of the genital tract of the dog: a mini review. Theriogenology 57: 1285-1291.

8. Gonzáles G 2004. Ecografía del aparato reproductor. En: Gobello C (ed). Temas de reproducción de caninos y felinos por autores latinoamericanos. Argentina: Gráfica Latina. p 27-42.

9. Guido MC. 2004. Ultra-sonografia do aparrelho reprodutor masculino. En: Carvalho CF (ed). Ultra-sonografia em pequenos animais. São Paulo: Roca. p 213-223.

10. Hörauf A, Thamke S. 2000. Specific diagnostic ultrasound procedures. In: Nautrup CP, Tobías R (eds). An atlas and textbook of diagnostic ultrasonography of the dog and cat. New Jersey, USA: Veterinary Learning System. p 366-395.

11. Johnston SD, Kamolpatana K, Kustritz R, Johnston GR. 2000. Prostatic disorders in the dog. Anim Reprod Sci 60-61: 405-415.

12. Kamolpatana K, Johnston, GR, Johnston SD. 2000. Determination of canine prostatic volume using transabdominal ultrasonography. Vet Radiol Ultrasound 41: 73-77.

13. Kanayama LM. 2004. Ultra-sonografia intervencionista. In: Carvalho CF (ed). Ultra-sonografia em pequenos animais. São Paulo: Roca. p 347-358.

14. Krawiec DR, Heflin D. 1992. Study of prostatic disease in dogs: 177 cases (1981-1986). J Am Vet Med Assoc 15: 1119-1122.
15. Maffrand CI, Gonzales G, Guendulain C, Forchetti O. 2007. Contribución de la citología al diagnóstico de las enfermedades prostáticas en el perro. Redvet 8: 1-14.

16. Morris J, Dobson J. 2002. Diagnóstico y estadificación. En: Oncología en pequeños animales. Buenos Aires: Ed Intermedica. p 13-26.

17. Mukaratirwa S, Chitura T. 2007. Canine subclinical prostatic disease: histological prevalence and validity of digital rectal examination as a screening test. J S Afr Vet Assoc 78: 66-68.

18. Nyland TG Mattoon JS, Herrgesell EJ, Wisner ER. 2004. Biopsia ecoguiada. En: Nyland TG, Mattoon JS (eds). Diagnóstico ecográfico en pequeños animales. Barcelona, España: Gráfica Multimédica. p 35-54.

19. Nyland TG Mattoon JS. 2004. Próstata y testículos. En: Diagnóstico ecográfico en pequeños animales. Barcelona, España: Gráfica Multimédica. p 260-276.

20. Ortega A, Acevedo M, Sauri C, Bolio M, Gutiérrez E. 2003. Prevalencia de tumor venéreo transmisible en perros callejeros de la ciudad de Mérida, Yucatán, México. Rev Biomed 14: 83-87.

21. Powe JR, Canfield PJ, Martin PA. 2004. Evaluation of the cytologic diagnosis of canine prostatic disorders. Vet Clin Pathol 33: 150-154.

22. Prüfer A, Luerssen D, Janthur M. 2000. Prostate gland. In: Nautrup CP, Tobías R (ed). An atlas and textbook of diagnostic ultrasonography of the dog and cat. New Jersey, USA: Veterinary Learning System. p 282-289.

23. Ruel Y, Barthez PY, Mailles A, Begon D. 1998. Ultrasonographic evaluation of the prostate in healthy intact dogs. Vet Radiol Ultrasound 39: 212-216.

24. Stornelli MA, Stornelli MC, Rodriguez RR, Scordellaro C, Savignone CA. 2002. Effect of finasteride on prostatic volumen in canine prostatic hypertrophy. Analecta Vet 22: 53-57. 
25. Swinney GR.1998. Prostatic neoplasia in five dogs. Aust Vet J 76: 699-674.

26. Teske E, Naan EC, Van Dijk, EM, Van Garden E. 2002. Canine prostate carcinoma: epidemiological evidence of an increased risk in castrated dogs. Mol Cell Endocrinol 197: 215-255.

27. Thomas G, Nyland TG, Wallack ST, Erik R, Wisner ER. 2002. Needle-tract implantation following us guided fineneedle aspiration biopsy of transitional cell carcinoma of the bladder, urethra, and prostate. Vet Radiol Ultrasound 43: 50-53.

28. Tyler RD, Cowell RL, Baldwin CJ, Morton RJ. 2003. Introducción. En: Cowell RL, Tyler RD, Meinkoth RH (eds). Citología y hematología diagnóstica en el perro y el gato. Barcelona, España: Multimédica. p 1-19.

29. White RAS. 2000. Prostatic surgery in the dog. Clin Tech Small Anim Pract 15: 46-51. 